\title{
Cyclic Operator Decomposition for Solving the Differential Equations
}

\author{
Ivan Gonoskov \\ Institute of Applied Physics of the Russian Academy of Sciences, Nizhny Novgorod, Russia \\ Email: ivan.gonoskov@gmail.com
}

Received October 19, 2012; revised November 23, 2012; accepted December 6, 2012

\begin{abstract}
We present an approach how to obtain solutions of arbitrary linear operator equation for unknown functions. The particular solution can be represented by the infinite operator series (Cyclic Operator Decomposition), which acts the generating function. The method allows us to choose the cyclic operators and corresponding generating function selectively, depending on initial problem for analytical or numerical study. Our approach includes, as a particular case, the perturbation theory, but generally does not require inside any small parameters and unperturbed solutions. We demonstrate the applicability of the method to the analysis of several differential equations in mathematical physics, namely, classical oscillator, Schrödinger equation, and wave equation in dispersive medium.
\end{abstract}

Keywords: Operator Decomposition; Spectral Theory; Propagator

\section{Introduction}

Various classical and quantum-mechanical problems in theoretical physics lead to the necessity of solving the linear operator equations for unknown functions and, in particular, the differential equations. Exact non-trivial analytical solutions of these equations, which include finite combinations of elementary operations and special functions, are known only for a number of specific cases. However, there are many actual and important cases for which such exact solutions were not still obtained even by using severe approximations for the corresponding interaction operators. For the cases when exact solutions are unknown, some approximate methods are usually used. They can be conventionally divided into two types: 1) varieties of perturbation theory and 2) numerical calculations (which generally are also based on perturbation theory). In spite of significant usefulness and applicability, these methods are not free from various limitations and disadvantages. The perturbation theory approaches may lead to divergent series, they need sometimes suitable unperturbed solutions, and, finally, they do not provide even estimations for precision in most cases (see $[1,2]$ and references therein). On the other hand, numerical schemes, which are from the very beginning approximate, usually also do not give reliable estimations for the precision (some reasonings can be found in [3,4]). Moreover, they can hardly give an asymptotic behavior of the solutions at infinity. Thus, the development of the general method which allows to overcome some of the above-mentioned difficulties is the main object of our study.

In this manuscript we develop an approach based on the theory of Cyclic Operator Decomposition (COD), which gives the opportunities to obtain solutions (exact or approximate) of the differential equations with arbitrary operators. The particular solution can be represented by the infinite cyclic operators series, which acts the previously determined generating function. The cyclic operators and the corresponding generating function (COD components) can be specified through the given operators in the differential equation. Under the convergence requirement, these COD components can be chosen in different ways depending on the certain problem statement. The procedure differs from the using of Born series (or corresponding Neumann series) in the perturbation theory [5-8] and S-matrix theory of Heisenberg, Feynmann and Dyson [9]. It can be understood easily by studying, for example, the difference between the formal definition of the generating function and Green's function (the last one is derived in some cases by using the operator resolvent formalism) [5,7,10-12]. Generally, the proposed series does not require any small parameters or unperturbed solutions for the convergence. But, as a matter of fact, the procedure can be transformed, under some certain choice of COD components, to the "standard" perturbation theory with small parameters. For the potentials without strong singularities, with reasonable 
choice of the cyclic operators and generating function, the corresponding series usually has uniform convergence. Some additional features and advantages of our approach for analytical and numerical solving the differential equations are demonstrated in sections below.

\section{Theory of Cyclic Operator Decomposition}

Let us start from the general case of operator equation for unknown function:

$$
\hat{D} \psi=0 \text {. }
$$

Here $\hat{D}$ is an arbitrary given linear operator and $\psi$ is an unknown function, which can be a vector or matrix of arbitrary dimensionality. This equation can lead in particular cases to arbitrary linear differential equations, which are considered in examples below.

Let us consider a pair of operators $\hat{G}$ and $\hat{V}$, which are determined by the following condition:

$$
\hat{D}=\hat{G}-\hat{V} \text {. }
$$

Since the choice of this pair is partly optional, we impose additional conditions on the operator $\hat{G}$ :

$$
\begin{gathered}
\exists \hat{G}^{-1} \text {, that: } \hat{G} \hat{G}^{-1}=\hat{I} ; \\
\exists \psi_{g} \equiv \equiv 0, \text { that: } \hat{G} \psi_{g} \equiv 0,
\end{gathered}
$$

where $\hat{I}$ is the identity operator. Any function $\psi_{g}$, which satisfies Equation (4), will be called generating function. Now we can write the following equation:

$$
\left(\hat{I}-\hat{G}^{-1} \hat{V}\right) \psi=\psi_{g}
$$

As we can check, under the above-mentioned conditions for $\hat{G}$ and $\psi_{g}$, any solution of Equation (5) fulfills Equation (1). Equation (5) can be solved in terms of the following Cyclic Operator Decomposition:

$$
\begin{aligned}
\psi & =\left[\hat{I}+\hat{G}^{-1} \hat{V}+\hat{G}^{-1} \hat{V} \hat{G}^{-1} \hat{V}+\cdots\right] \psi_{g} \\
& =\left[\hat{I}+\sum_{n=1}^{\infty}\left(\hat{G}^{-1} \hat{V}\right)^{n}\right] \psi_{g} .
\end{aligned}
$$

This is the exact particular solution of Equation (1) with corresponding particular COD components determined by Equations (2) and (3). The solution makes sense only if the obtained series is convergent. This can be achieved in different cases depending on $\hat{G}^{-1} \hat{V}$ and $\psi_{g}$, for example, if we work in Banach space and corresponding operator norm is $\left\|\hat{G}^{-1} \hat{V}\right\|<1$. The convergence of some similar operator series was considered also in $[5,13]$.

The theory can be easily generalized also to the case of the equations with given sources:

$$
\hat{D} \psi=\varphi
$$

where an arbitrary given function $\varphi$ describes the arbitrary sources. The unknown function $\psi$ could be found naturally if the inverse operator is known: $\psi=\hat{D}^{-1} \varphi$. However, the inverse operator $\hat{D}^{-1}$ can not be easily found for a number of problems. Then, we can write a solution of this equation analogously by using COD:

$$
\psi=\left[\hat{I}+\sum_{n=1}^{\infty}\left(\hat{G}^{-1} \hat{V}\right)^{n}\right]\left(\psi_{g}+\hat{G}^{-1} \varphi\right) .
$$

In contrast to the case of Equation (1), now we can choose $\psi_{g} \equiv 0$ for some non-trivial particular solutions. Then we can obtain the particular solution of Equation (7), which corresponds to the following particular determination of the inverse operator in terms of COD:

$$
\hat{D}^{-1}=\left[I+\sum_{n=1}^{\infty}\left(\hat{G}^{-1} \hat{V}\right)^{n}\right] \hat{G}^{-1} .
$$

Important feature of the proposed theory is that, while the conditions Equations (3) and (4) should be fulfilled and the convergence of the series is necessary, we still have a great freedom of choosing $\hat{G}$ and corresponding $\psi_{g}$. Generally, it gives us opportunities to obtain all the possible solutions of Equation (1). Sometimes we can naturally choose $\hat{G}$ and $\psi_{g}$ in accordance, for example, with the corresponding initial conditions for Cauchy problem or boundary conditions for boundary-value problems.

In some cases, the exact solution Equation (6) can be used naturally for obtaining the approximate solution with finite number of terms. It can be done, for example, when, starting from certain number $n$, the following conditions are satisfied: $\left\|\left(\hat{G}^{-1} \hat{V}\right)^{n} \psi_{g}\right\| \gg\left\|\left(\hat{G}^{-1} \hat{V}\right)^{n+1} \psi_{g}\right\| \gg \cdots$. These are the sufficient conditions enabling one to derive the approximate solution with the prescribed accuracy. Further, the proposed method provides another advantage if one performs numerical calculations. According to the exact solution Equation (6), we can use recurrent relations when calculating numerically the approximate solutions. In this case, the calculation of any next term in the corresponding series does not require more numerical resources than the calculation of the previous one.

\section{Examples}

In this section we apply the proposed theory of Cyclic Operator Decomposition for the various cases of differential equations. Let us first consider the Cauchy problem for the equation of classical oscillator. Note that this equation, if written in other variables, is the stationary one-dimensional Schrödinger equation with given energy, and it can be transformed also to the Riccati equation by using logarithmic substitution. 


$$
\begin{aligned}
& \ddot{f}+\omega^{2}(t) f=0, \\
& f\left(t_{a}\right)=a, \\
& \dot{f}\left(t_{b}\right)=b,
\end{aligned}
$$

where $f(t)$ is an unknown function, $\omega^{2}(t)$ is an arbitrary time-dependent frequency and $a, b$ are arbitrary constants. Here, it is natural to choose the components for COD as follows:

$$
\begin{aligned}
& \hat{G}=\frac{\mathrm{d}^{2}}{\mathrm{~d} t^{2}}, \psi_{g}=a+b\left(t-t_{a}\right), \\
& \hat{G}^{-1}=\int_{t_{a}}^{t} \mathrm{~d} \tau_{1} \int_{t_{b}}^{\tau_{1}} \mathrm{~d} \tau_{2}, \hat{V}=-\omega^{2}(t) .
\end{aligned}
$$

If we fix (by our local convention, which we will use below) that we write for brevity the same variable upper limit of integration as the integration variable and determine the successive integration (step by step from right to left), we can write a simple expression for the solution:

$$
\begin{aligned}
f(t)= & {\left[a+b\left(t-t_{a}\right)\right]-\int_{t_{a}}^{t} \mathrm{~d} t \int_{t_{b}}^{t} \omega^{2}(t)\left[a+b\left(t-t_{a}\right)\right] \mathrm{d} t } \\
& +\int_{t_{a}}^{t} \mathrm{~d} t \int_{t_{b}}^{t} \omega^{2}(t) \mathrm{d} t \int_{t_{a}}^{t} \mathrm{~d} t \int_{t_{b}}^{t} \omega^{2}(t)\left[a+b\left(t-t_{a}\right)\right] \mathrm{d} t-\cdots
\end{aligned}
$$

It is important to note now, that the presented series (Equation (12)) has rapid uniform convergence at least in any interval, where $\omega^{2}(t)$ is bounded. For example, in the limited interval $[0, t]$ the rate of convergence for $f(t)$ can be estimated in the following way (we assume here for simplicity, that $t_{a}=t_{b}=0, b=0$, and the maximum of $\left|\omega^{2}(t)\right|$ in the corresponding interval is $\left.C_{\max }\right)$ :

$$
(n \text {-th term of series }) \leq|a| \frac{\left(C_{\max }\right)^{n} t^{2 n}}{(2 n) !} .
$$

In the same way, the convergence can be demonstrated for other different COD's, when the cyclic operators are bounded for the given generating functions in the given relevant interval. Moreover, if additionally $\omega(t)$ is a real function, $\omega^{2}(t)>\omega^{2}(0) \geq 0$ and $t>0$, we have a decreasing alternating series for the above example, and we can estimate the precision of partial sum of the series by the value of the last term.

Now we focus on some particular cases of $\omega^{2}(t)$. To demonstrate that it is possible to obtain a solution with any prescribed precision, we consider a case when

$$
\omega^{2}(t)=\left[1-\frac{1}{2} \sin t\right]>0
$$

and $t_{a}=t_{b}=0, a=1, b=0$. Calculation of the first two terms in Equation (12) gives

$$
f(t)=\left[1-\frac{1}{2}\left(t^{2}-t+\sin t\right)\right]+\delta .
$$

By calculating the third term in Equation (12), we obtain $\delta<0.0273$ if we consider $t$ in the interval $[0,1]$.

Sometimes we can find also the asymptotic behavior of the solution. As an example, we consider the case $\omega^{2}(t)=-t^{\alpha}$ and $t_{a}=t_{b}=0, a=1, b=0$, where $\alpha$ is an arbitrary constant, $\alpha>-1$. Using again Equation (12) we obtain exact solution in the following form:

$$
\begin{aligned}
f(t)= & +\frac{t^{\alpha+2}}{(\alpha+1)(\alpha+2)} \\
& +\frac{t^{2 \alpha+4}}{(\alpha+1)(\alpha+2)(2 \alpha+3)(2 \alpha+4)}+\cdots
\end{aligned}
$$

By analyzing the corresponding series we can obtain a simple upper estimate for the solution $(\forall t>0)$ :

$$
f(t)<1+\frac{t^{\alpha+2}}{(\alpha+1)(\alpha+2)} \cdot \exp \left[\frac{2 t^{\frac{1}{2} \alpha+1}}{\alpha+2}\right]
$$

which gives us the following asymptotic behavior at $t \rightarrow \infty$ :

$$
f(t) \propto \exp \left[\frac{2 t^{\frac{1}{2} \alpha+1}}{\alpha+2}\right] .
$$

In this case, the same asymptotic can be found also from WKB theory (see, for example, quasiclassical approximation in $[14,15])$.

Let us now demonstrate the selective choice of cyclic operators. For that we consider stationary one-dimensional Schrödinger equation (we use below the units where $\left.\hbar=1, m_{p}=1\right)$ :

$$
\left[2 E+\frac{\mathrm{d}^{2}}{\mathrm{~d} x^{2}}-A \cdot \mathrm{e}^{\beta x}\right] \psi(x)=0
$$

where $\psi(x)$ is an unknown function, which describes quantum state with energy $E$ in the continuum; $A, \beta$ are arbitrary real constants. Without loss of generality (one can use scale transformations of Equation (18)) we can assume $2 E=m^{2}, \beta=1$. To find the solution of this equation, we can choose the components for COD in different ways. For example, if one interests in the behavior of $\psi(x)$ near $x=0$ and in small values of $E$, he can choose $\hat{G}=\frac{\mathrm{d}^{2}}{\mathrm{~d} x^{2}}$ and use nearly the same technique as in Equations (11) and (12). However, this choice can be inconvenient for the analysis of the long-range behavior. Another variant of choosing the components for COD is the following (we use also Equation (9) for the particular determination of $\hat{G}^{-1}$ ): 


$$
\begin{aligned}
& \hat{G}=m^{2}+\frac{\mathrm{d}^{2}}{\mathrm{~d} x^{2}}, \psi_{g}(x)=C_{1} \mathrm{e}^{i m x}+C_{2} \mathrm{e}^{-i m x}, \\
& \hat{G}^{-1}=\left[\hat{1}+\sum_{k=1}^{\infty}\left(\hat{G}_{0}^{-1} \hat{V}_{0}\right)^{k}\right] \hat{G}_{0}^{-1}, \\
& \hat{G}_{0}^{-1}=\int_{-\infty}^{x} \mathrm{~d} x \int_{-\infty}^{x} \mathrm{~d} x, \hat{V}_{0}=-m^{2}, \hat{V}=A \cdot \mathrm{e}^{x},
\end{aligned}
$$

where $C_{1}$ and $C_{2}$ are arbitrary constants. To obtain the general solution, we consider the particular case of generating function $\tilde{\psi}_{g}(x)=\mathrm{e}^{i m x}$ and corresponding particular solution $\psi_{p}(x)$. Then we derive by using a rule of infinite geometric series:

$$
\begin{aligned}
\hat{G}^{-1} \hat{V} \tilde{\psi}_{g}(x) & =\left[\hat{1}+\sum_{K=1}^{\infty}\left(\hat{G}_{0}^{-1} \hat{V}_{0}\right)^{k}\right] \frac{A \cdot \mathrm{e}^{(1+i m) x}}{(1+i m)^{2}} \\
& =\left[1+\sum_{K=1}^{\infty}\left(\frac{m^{2}}{(1+i m)^{2}}\right)^{k}\right] \frac{A \cdot \mathrm{e}^{(1+i m) x}}{(1+i m)^{2}} \\
& =\frac{A \cdot \mathrm{e}^{(1+i m) x}}{(1+2 i m)} .
\end{aligned}
$$

From here we obtain

$$
\psi_{p}(x)=\mathrm{e}^{i m x}\left[1+\sum_{n=1}^{\infty} A^{n} \mathrm{e}^{n x} \cdot \prod_{k=1}^{n} \frac{1}{\left(k^{2}+2 i m k\right)}\right]
$$

and the general solution in the following form:

$$
\psi(x)=C_{1} \cdot \psi_{p}(x)+C_{2} \cdot \psi_{p}^{*}(x)
$$

The corresponding series converges at any $A$ and real $m$.

In a similar way we can obtain solutions for multidimensional equations. Let us consider the stationary Schrödinger equation with potential surface $U(\boldsymbol{r})$, multidimensional Laplace operator $\Delta$, and energy $E$ :

$$
[\Delta+2(E-U(\boldsymbol{r}))] \psi(\boldsymbol{r})=0 \text {. }
$$

Then we can choose $\hat{G}=\Delta, \hat{V}=-2(E-U(\boldsymbol{r}))$, and $\psi_{g}$ is any solution of $\Delta \psi_{g}=0$. From corresponding COD we can obtain a solution:

$$
\psi(\boldsymbol{r})=\left[1+\Delta^{-1} V+\Delta^{-1} V \Delta^{-1} V+\cdots\right] \psi_{g}=0 .
$$

The inverse Laplace operator can be written, for example, as $\Delta^{-1}=-\hat{F}^{-1} k^{-2} \hat{F}$, where $\hat{F}$ is the Fourier transform operator and $k$ is an absolute value of the wave vector in this transform.

Another choice of COD components can be better for the finding of the bound states with $E<0$. We can choose: $\hat{G}=2 E+\Delta, \hat{V}=2 U(\boldsymbol{r}), \psi_{g}$ is any solution of $[2 E+\Delta] \psi_{g}=0$, and write the inverse operator $\hat{G}^{-1}$ for COD as follows:

$$
\hat{G}^{-1}=(2 E+\Delta)^{-1}=\hat{F}^{-1} \frac{1}{2 E-k^{2}} \hat{F} .
$$

In this way, we can calculate in some cases the terms in the corresponding COD by evaluating the poles at imaginary values $k_{P}= \pm i \sqrt{-2 E}$.

Now we consider time-dependent three-dimensional Schrödinger equation to demonstrate other applications of the proposed method. Let us consider propagation of charged particle with arbitrary electromagnetic interactions (below $\boldsymbol{A}(\boldsymbol{r}, t)$ is the vector potential, and $q=c=1$ ):

$$
\left[i \frac{\partial}{\partial t}-\frac{1}{2}(i \nabla+\boldsymbol{A}(\boldsymbol{r}, t))^{2}-U(\boldsymbol{r}, t)\right] \psi(\boldsymbol{r}, t)=0 .
$$

Here, we can choose the components for COD in a variety of ways depending on peculiar properties of the interactions. One special choice is the following:

$$
\begin{aligned}
& \hat{G}=i \frac{\partial}{\partial t}, \psi_{g}=\psi\left(\boldsymbol{r}, t_{0}\right)=\psi_{0}(\boldsymbol{r}), \\
& \hat{G}^{-1}=-i \int_{t_{0}}^{t} \mathrm{~d} t \\
& \hat{V}=\frac{1}{2}(i \nabla+\boldsymbol{A}(\boldsymbol{r}, t))^{2}+U(\boldsymbol{r}, t),
\end{aligned}
$$

where $\hat{V}$ corresponds to the time-dependent Hamiltonian. It gives the following solution:

$$
\psi(\boldsymbol{r}, t)=\left[1+(-i) \int_{t_{0}}^{t} \mathrm{~d} t \hat{V}+(-i)^{2} \int_{t_{0}}^{t} \mathrm{~d} t \hat{V} \int_{t_{0}}^{t} \mathrm{~d} t \hat{V}+\cdots\right] \psi_{0}(\boldsymbol{r}) .
$$

This solution can be useful for the numerical calculations, namely, for the finding the propagator $\hat{P}$, which gives: $\psi(\boldsymbol{r}, t+\Delta t)=\hat{P} \psi(\boldsymbol{r}, t)+O\left(\Delta t^{n}\right)$, see also [4] and references therein. If we use additionally internal timeordering, we can transform this expression to Dyson series (see $[9,16])$.

Finally, we consider the wave equation for electromagnetic waves in dispersive medium. We assume that an arbitrarily given operator $\hat{\epsilon}(\boldsymbol{r}, t)$ which describes electric dispersion does not depend nonlinearly on the field, i.e. we still have linear problem. In this case the equation for unknown vector potential $\boldsymbol{A}(\boldsymbol{r}, t)$ is the following (see for example [17]):

$$
\left[\frac{\partial}{\partial t}\left(\hat{\epsilon}(\boldsymbol{r}, t) \frac{\partial}{\partial t}\right)-\nabla^{2}\right] \boldsymbol{A}(\boldsymbol{r}, t)=0
$$

with the initial conditions

$$
\boldsymbol{A}_{(t=0)}=\boldsymbol{S}(\boldsymbol{r}),\left(\frac{\partial \boldsymbol{A}}{\partial t}\right)_{(t=0)}=\boldsymbol{R}(\boldsymbol{r}) .
$$

We can choose the following components for COD: 


$$
\begin{aligned}
& \hat{G}=\frac{\partial}{\partial t}\left(\hat{\epsilon}(\boldsymbol{r}, t) \frac{\partial}{\partial t}\right), \\
& \psi_{g}=\boldsymbol{S}(\boldsymbol{r})+\int_{t_{0}}^{t} \mathrm{~d} t \hat{\epsilon}^{-1}(\boldsymbol{r}, t) \boldsymbol{R}(\boldsymbol{r}), \\
& \hat{G}^{-1}=\int_{t_{0}}^{t} \mathrm{~d} t \hat{\epsilon}^{-1}(\boldsymbol{r}, t) \int_{t_{0}}^{t} \mathrm{~d} t, \hat{V}=\nabla^{2}
\end{aligned}
$$

Then, we can find the solution, which follows from the corresponding COD series:

$$
\begin{aligned}
\boldsymbol{A}(\boldsymbol{r}, t)= & {\left[1+\sum_{n=1}^{\infty}\left(\int_{t_{0}}^{t} \mathrm{~d} t \hat{\epsilon}^{-1}(\boldsymbol{r}, t) \int_{t_{0}}^{t} \mathrm{~d} t \nabla^{2}\right)^{n}\right] } \\
& \times\left[\boldsymbol{S}(\boldsymbol{r})+\int_{t_{0}}^{t} \mathrm{~d} t \hat{\epsilon}^{-1}(\boldsymbol{r}, t) \boldsymbol{R}(\boldsymbol{r})\right] .
\end{aligned}
$$

For this solution the initial magnetic field is equal to $[\nabla \times \boldsymbol{S}(\boldsymbol{r})]$ and the initial electric field is equal to $-\boldsymbol{R}(\boldsymbol{r})$.

\section{Conclusion}

In summary, we propose the theory of Cyclic Operator Decomposition, which allows one to obtain particular solutions of linear operator equations for unknown functions. In most cases it is possible to obtain all the possible solutions, which satisfy the given conditions. We demonstrate by some reasonings and particular examples that our approach has the following remarkable properties: 1) there is a freedom in choosing the COD components depending on the certain problem; 2) there is a rapid uniform convergence for most of the considered cases; 3 ) it is possible to find the asymptotic behavior of the solutions; 4) in many cases when one is analyzing the approximate solution, it is possible to estimate the accuracy; 5) the proposed approach gives good opportunities for efficient implementation of numerical calculations due to the recurrent relations that can be used in COD.

\section{Acknowledgements}

Author would like to thank academician L. D. Faddeev, M. Yu. Emelin, M. Yu. Ryabikin, and A. A. Gonoskov for the useful and stimulating discussions.

\section{REFERENCES}

[1] F. J. Dyson, "Divergence of Perturbation Theory in Quantum Electrodynamics," Physical Review, Vol. 85, No. 4, 1952, pp. 631-632. doi:10.1103/PhysRev.85.631

[2] A. V. Turbiner, "The Eigenvalue Spectrum in Quantum Mechanics and the Nonlinearization Procedure," Soviet Phy- sics Uspekhi, Vol. 27, No. 9, 1984, p. 668. doi:10.1070/PU1984v027n09ABEH004155

[3] J. Fleck, J. Morris and M. Feit, "Time-Dependent Propagation of High Energy Laser Beams through the Atmosphere," Applied Physics A: Materials Science and Processing, Vol. 10, No. 2, 1976, pp. 129-160. doi:10.1007/BF00896333

[4] A. N. Drozdov and S. Hayashi, "Numerical Test of Approximate Single-Step Propagators: Harmonic Power Series Expansions versus System-Specific Split Operator Representations," Physical Review E, Vol. 59, No. 2, 1999, pp. 1386-1397. doi:10.1103/PhysRevE.59.1386

[5] J. V. Corbett and J. Math, "Convergence of the Born Series," Journal of Mathematical Physics, Vol. 9, No. 6, 1968, p. 891. doi:10.1063/1.1664655

[6] M. Wellner, "Improvement of the Born Series at Low Energy," Physical Review, Vol. 132, No. 4, 1963, pp. 18481853. doi:10.1103/PhysRev.132.1848

[7] R. Perez and J. Math, "On the Expansion of the Propagator in Power Series of the Coupling Constant," Journal of Mathematical Physics, Vol. 20, No. 2, 1979, p. 241. doi: $10.1063 / 1.524070$

[8] F. S. Bemfica and H. O. Girotti, "Born Series and Unitarity in Noncommutative Quantum Mechanicsphys," Physical Review D, Vol. 77, No. 2, 2008, Article ID: 027704. doi:10.1103/PhysRevD.77.027704

[9] F. J. Dyson, "The S Matrix in Quantum Electrodynamics," Physical Review, Vol. 75, No. 11, 1949, pp. 1736 1755. doi:10.1103/PhysRev.75.1736

[10] J. H. Eberly and H. R. Reiss, "Electron Self-Energy in Intense Plane-Wave Field," Physical Review, Vol. 145, No. 4, 1966, pp. 1035-1040. doi:10.1103/PhysRev.145.1035

[11] H. R. Reiss and J. H. Eberly, "Green's Function in Intense-Field Electrodynamics," Physical Review, Vol. 151, No. 4, 1966, pp. 1058-1066. doi:10.1103/PhysRev.151.1058

[12] A. Mockel and J. Math, "Invariant Imbedding as a Generalization of the Resolvent Equation," Journal of Mathematical Physics, Vol. 8, No. 12, 1967, p. 2318. doi:10.1063/1.1705158

[13] B. Curgus and T. T. Read, "Discreteness of the Spectrum of Second-Order Differential Operators and Associated Embedding Theorems," Journal of Differential Equations, Vol. 184, No. 2, 2002, pp. 526-548. doi:10.1006/jdeq.2001.4152

[14] L. Landau and E. Lifshitz, "Quantum Mechanics Non-Relativistic Theory," 3rd Edition, Pergamon Press, Oxford, 1977.

[15] L. Schiff, "Quantum Mechanics,” 3rd Edition, McGraw Hill, New York, 1968.

[16] F. J. Dyson, "The Schrödinger Equation in Quantum Electrodynamics," Physical Review, Vol. 83, No. 6, 1951, pp. 1207-1216.

[17] L. Landau and E. Lifshitz, "Electrodynamics of Continuous Media,” Vol. 8, Butterworth-Heinemann, Oxford, 1984. 